\title{
MAE Regimen
}

National Cancer Institute

\section{Source}

National Cancer Institute. MAE Regimen. NCI Thesaurus. Code C67485.

A regimen consisting of cytarabine (Ara-C), mitoxantrone and etoposide, used as an induction treatment for pediatric acute myeloid leukemia (AML). 